Supporting Information for

\title{
Asymmetric Benzoin Condensation Catalyzed by Chiral Rotaxanes \\ Tethering Thiazolium Salt Moiety via The Cooperation of The Component: \\ Can Rotaxane Be An Effective Reaction Field?
}

\author{
Yuya Tachibana, ${ }^{\dagger}$ Nobuhiro Kihara ${ }^{\ddagger}$, and Toshikazu Takata ${ }^{*} \dagger$ \\ ${ }^{\dagger}$ Department of Organic and Polymeric Materials, Tokyo Institute of Technology, \\ Ookayama, Meguro, Tokyo 152-8552, Japan \\ and Department of Applied Chemistry, Graduate School of Engineering, \\ Osaka Prefecture University, Sakai, Osaka 599-8531, Japan
}


Methods and Materials. $\quad{ }^{1} \mathrm{H}$ and ${ }^{13} \mathrm{C}$ NMR spectra were recorded on a JEOL GSX500 (500 MHz) spectrometers using tetramethylsilane as an internal standard. IR spectra were recorded on a JASCO FT/IR-460plus spectrometer. FAB-MS analyses were made on a FINNIGAN MAT TSQ-70 instrument. Melting points were measured on a Yanako MP-3 instrument. Optical rotations were measured on a JASCO DIP-1000 instrument. Preparative GPC was carried out using JAI LC-908 equipped with JAIGEL-1C and JAIGEL-2C columns. Enantiomeric excess (e.e.) was measured on a JASCO GULLIVER system equipped with DAICEL CHIRALPAK AD-H column. Acetonitrile, $N, N$-dimethylformamide, triethylamine, and all aldehydes were used after distillation over calcium hydride. Methanol was distilled over magnesium before use. Dichloromethane was treated with phosphorous pentoxide before distillation over calcium hydride. Other chemicals were reagent grade and used without further purification.

General procedure for benzoin condensation. To a solution of triethylamine and an aldehyde was added a chiral thiazolium salt under an argon atmosphere. The reaction mixture was allowed to stand at mentioned temperature. After $24 \mathrm{~h}$, the reaction mixture was evaporated to give the crude solid, which was purified by silica gel column chromatography (eluent: dichloromethane). The product was identified as benzoin by ${ }^{1} \mathrm{H}$ NMR spectra. ${ }^{1}$ 


\section{Scheme S1}

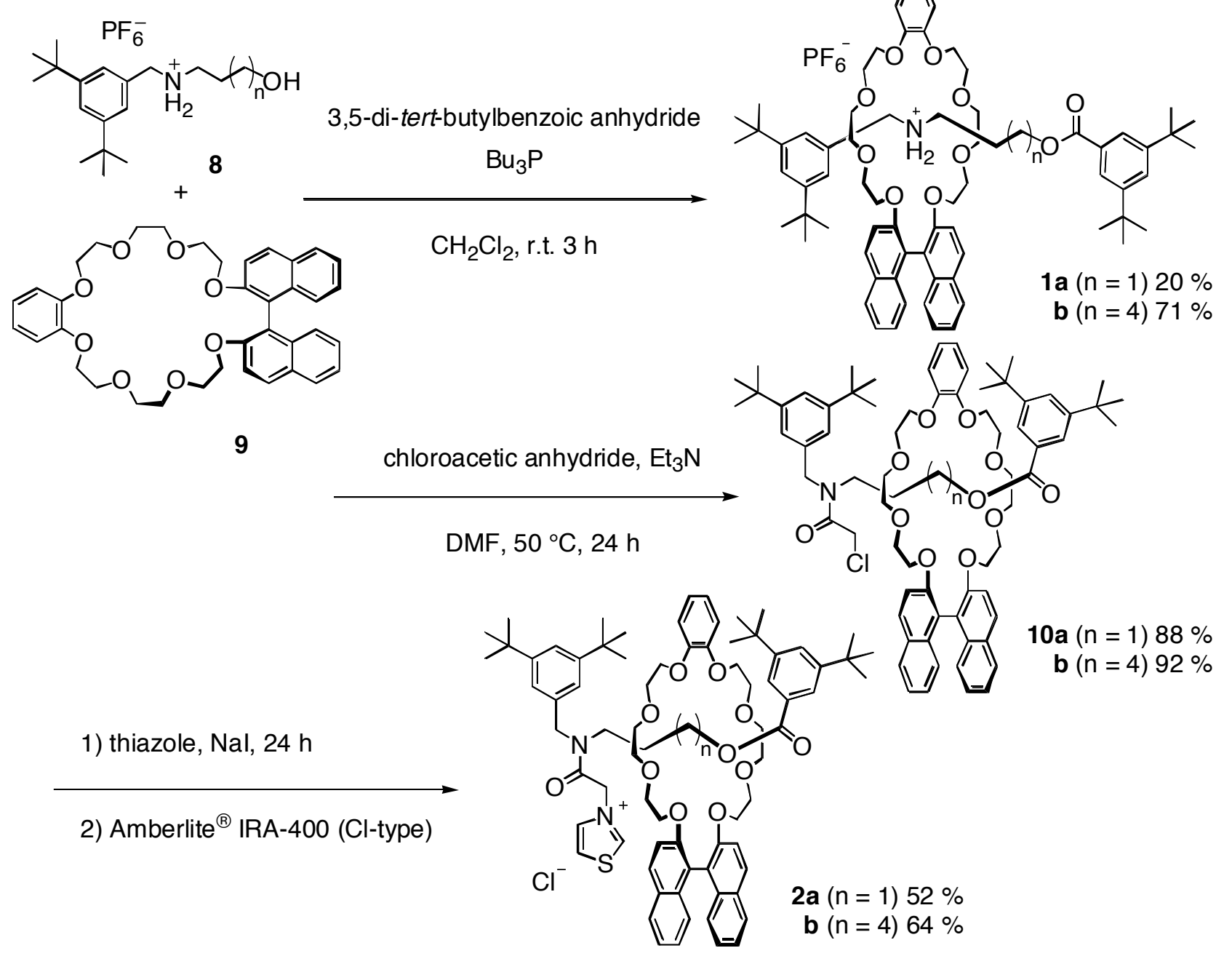

\section{(R)-Chiral rotaxane (1). General procedure.}

To a solution of ammonium salt $8(0.70 \mathrm{mmol})$ and chiral crown ether $9(0.70 \mathrm{mmol})$ in $\mathrm{CH}_{2} \mathrm{Cl}_{2}(1.4 \mathrm{~mL})$ were added 3,5-di-tert-butylbenzoic anhydride $(0.84 \mathrm{mmol})$ and tributylphosphine $(0.07 \mathrm{mmol})$. After the reaction mixture was allowed to stand at room temperature for $6 \mathrm{~h}$, the mixture was washed with $1 \mathrm{M}$ hydrochloric acid, $5 \%$ sodium carbonate, then brine, dried over anhydrous magnesium sulfate, and evaporated in vacuo to give a crude product, which was purified by preparative HPLC (eluent: chloroform).

1a: amorphous solid. yield $20 \%$. m.p. $96^{\circ} \mathrm{C}$.

${ }^{1} \mathrm{H}$ NMR (500 MHz, $\left.\mathrm{CDCl}_{3}\right) \delta 7.87(\mathrm{~d}, J=2.0 \mathrm{~Hz}, 2 \mathrm{H}), 7.84(\mathrm{~d}, J=8.5 \mathrm{~Hz}, 2 \mathrm{H}), 7.81-7.78(\mathrm{~m}, 2 \mathrm{H}), 7.69(\mathrm{~s}, 1 \mathrm{H})$, $7.55(\mathrm{~d}, J=8.5 \mathrm{~Hz}, 1 \mathrm{H}), 7.37-7.33(\mathrm{~m}, 3 \mathrm{H}), 7.26-7.13(\mathrm{~m}, 4 \mathrm{H}), 7.09(\mathrm{br}, 2 \mathrm{H}), 7.04(\mathrm{~s}, 2 \mathrm{H}), 6.94(\mathrm{~d}, J=8.5 \mathrm{~Hz}$, $1 \mathrm{H})$, 6.86-6.81 (m, 3H), 6.76-6.74 (m, 1H), 4.32-4.10 (m, 7H), 4.04-4.00 (m, 2H), 3.77-3.67 (m, 3H), 3.63-3.49 (m, 5H), 3.39-3.17 (m, 10H), 3.03-2.93 (m, 3H), 2.00-1.85 (m, 2H), 1.37 (s, 18H), 1.17 (s, 18H) ppm.

${ }^{13} \mathrm{C}$ NMR $\left(125 \mathrm{MHz}, \mathrm{CDCl}_{3}\right) \delta 166.83,154.48,154.15,151.15,146.66,146.33,133.59,133.55,130.85,130.00$, 129.94, 129.77, 129.66, 129.16, 127.97, 127.31, 126.51, 126.47, 125.08, 124.94, 124.41, 124.22, 124.09, 123.63, $123.34,121.85,121.77,120.97,118.12,116.65,112.42,112.20,71.23,71.00,70.82,70.71,70.47,70.07,69.69$, 69.37, 68.28, 67.71, 61.44, 52.96, 45.96, 35.01, 34.76, 31.46, 31.33, $26.11 \mathrm{ppm}$.

IR (KBr): $2962\left(v_{\mathrm{C}-\mathrm{H}}\right), 1717\left(v_{\mathrm{C}=\mathrm{O}, \text { ester }}\right), 1240\left(v_{\mathrm{C}-\mathrm{O}-\mathrm{CO}, \text { as }}\right), 1129\left(v_{\mathrm{C}-\mathrm{O}-\mathrm{CO}, \mathrm{s}}\right), 843\left(v_{\mathrm{P}-\mathrm{F}, \text { as }}\right), 557\left(v_{\mathrm{P}-\mathrm{F}, \mathrm{s}}\right) \mathrm{cm}^{-1}$.

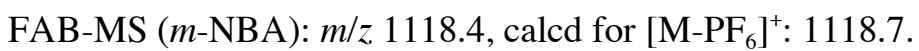

$[\alpha]_{\mathrm{D}}^{27} 105.4^{\circ}\left(\mathrm{c}=0.100, \mathrm{CH}_{2} \mathrm{Cl}_{2}\right)$.

Anal, calcd for $\mathrm{C}_{71} \mathrm{H}_{92} \mathrm{~F}_{6} \mathrm{NO}_{10} \mathrm{P} \cdot \mathrm{H}_{2} \mathrm{O}: \mathrm{C}, 66.49 ; \mathrm{H}, 7.39 ; \mathrm{N}, 1.09$. found: $\mathrm{C}, 66.33 ; \mathrm{H}, 7.41 ; \mathrm{N}, 1.24$. 
1b: amorphous solid. yield $71 \%$. m.p. $86-88^{\circ} \mathrm{C}$.

${ }^{1} \mathrm{H}$ NMR $\left(500 \mathrm{MHz}, \mathrm{CDCl}_{3}\right) \delta 8.00(\mathrm{~d}, J=9.0 \mathrm{~Hz}, 1 \mathrm{H}), 7.91-7.85(\mathrm{~m}, 5 \mathrm{H}), 7.65(\mathrm{~s}, 1 \mathrm{H}), 7.57(\mathrm{~d}, J=9.5 \mathrm{~Hz}, 1 \mathrm{H})$, 7.39-7.34 (m, 3H), 7.26-7.15 (m, 7H), 6.99 (br, 2H), 6.88-6.85 (m, 4H), 4.26-4.14 (m, 8H), 4.10-4.05 (m, 1H), 4.02-3.99 (m, 1H), 3.93-3.90 (m, 1H), 3.77-3.74 (m, 1H), 3.67-3.30 (m, 14H), 3.23-3.10 (m, 3H), 2.95-2.91 (m, $1 \mathrm{H}), 2.82$ (br, 1H), 2.57 (br, 1H), 1.58-1.52 (m, 2H), $1.36(\mathrm{~s}, 18 \mathrm{H}), 1.20(\mathrm{~s}, 18 \mathrm{H}), 1.16-1.07$ (m, 2H), 1.01-0.96 (m, 2H) $\mathrm{ppm}$.

${ }^{13} \mathrm{C}$ NMR $\left(125 \mathrm{MHz}, \mathrm{CDCl}_{3}\right) \delta 167.07,154.77,154.29,151.15,150.92,146.82,146.58,133.62,133.49,130.93$, $129.97,129.90,129.87,129.79,129.67,128.02,127.92,126.96,126.53,126.47,124.99,124.97,124.37,124.30$, $123.54,123.29,121.85,121.77,121.66,121.18,118.19,116.98,112.45,112.38,71.53,70.95,70.82,70.76$, $70.567,70.33,70.16,69.62,69.16,68.26,68.11,64.40,53.00,48.83,34.94,34.78,31.39,31.32,28.44,26.13$, $25.99,25.33 \mathrm{ppm}$.

IR (KBr): $2961\left(v_{\mathrm{C}-\mathrm{H}}\right), 1714\left(v_{\mathrm{C}=\mathrm{O}, \text { ester }}\right), 1246\left(v_{\mathrm{C}-\mathrm{O}-\mathrm{CO}, \text { as }}\right), 1129\left(v_{\mathrm{C}-\mathrm{O}-\mathrm{CO}, \mathrm{s}}\right), 844\left(v_{\mathrm{P}-\mathrm{F}, \text { as }}\right), 558\left(v_{\mathrm{P}-\mathrm{F}, \mathrm{s}}\right) \mathrm{cm}^{-1}$.

FAB-MS ( $m$-NBA): $m / z 1160.7$, calcd for $\left[\mathrm{M}^{-\mathrm{PF}_{6}}\right]^{+}: 1160.7$.

$[\alpha]_{\mathrm{D}}^{27} 98.0^{\circ}\left(\mathrm{c}=0.100, \mathrm{CH}_{2} \mathrm{Cl}_{2}\right)$

Anal, calcd for $\mathrm{C}_{74} \mathrm{H}_{98} \mathrm{~F}_{6} \mathrm{NO}_{10} \mathrm{P} \cdot \mathrm{H}_{2} \mathrm{O}: \mathrm{C}, 67.10 ; \mathrm{H}, 7.61 ; \mathrm{N}, 1.06$. found: $\mathrm{C}, 67.47 ; \mathrm{H}, 7.75 ; \mathrm{N}, 1.20$.

\section{$(R)$ - $N$-Chloroacetyl rotaxane (10). General procedure.}

A solution of $1(0.131 \mathrm{mmol})$, chloroacetic anhydride $(1.31 \mathrm{mmol})$, and triethylamine $(0.66 \mathrm{mmol})$ in $N, N$ dimethylformamide $(2.0 \mathrm{~mL})$ was stirred at $50{ }^{\circ} \mathrm{C}$ for $24 \mathrm{~h}$. After addition of aquaous $5 \%$ sodium carbonate, solution, the mixture was extracted with ethyl acetate. The organic layer was washed with a mixture of $5 \%$ sodium carbonate solution and methanol $(\mathrm{v} / \mathrm{v}=1 / 1), 2 \mathrm{M}$ hydrochloric acid, then brine, dried over anhydrous magnesium sulfate, and evaporated in vacuo to give a crude product, which was purified by preparative HPLC (eluent: chloroform).

10a: amorphous solid. yield $88 \%$. m.p. $115-117^{\circ} \mathrm{C}$.

${ }^{1} \mathrm{H} \mathrm{NMR}\left(500 \mathrm{MHz}, \mathrm{CDCl}_{3}\right) \delta 7.99$ (d, $\left.J=9.0 \mathrm{~Hz}, 1 \mathrm{H}\right), 7.88(\mathrm{~d}, J=8.0 \mathrm{~Hz}, 1 \mathrm{H}), 7.83$ (d, $\left.J=1.5 \mathrm{~Hz}, 2 \mathrm{H}\right), 7.67$ (s, $1 \mathrm{H}), 7.50(\mathrm{~d}, J=8.5 \mathrm{~Hz}, 1 \mathrm{H}), 7.44(\mathrm{~d}, J=8.0 \mathrm{~Hz}, 1 \mathrm{H}), 7.33-7.30(\mathrm{~m}, 1 \mathrm{H}), 7.24-7.15(\mathrm{~m}, 7 \mathrm{H}), 7.07(\mathrm{~s}, 1 \mathrm{H}), 6.85-$ $6.77(\mathrm{~m}, 4 \mathrm{H}), 6.30(\mathrm{~s}, 2 \mathrm{H}), 4.75(\mathrm{~d}, J=16.5 \mathrm{~Hz}, 1 \mathrm{H}), 4.62-4.57(\mathrm{~m}, 1 \mathrm{H}), 4.36-4.29(\mathrm{~m}, 4 \mathrm{H}), 4.20-4.15(\mathrm{~m}, 2 \mathrm{H})$, 4.10-4.02 (m, 3H), 3.95-3.92 (m, 2H), 3.84-3.80 (m, 1H), 3.72-3.60 (m, 4H), 3.54-3.22 (m, 9H), 3.10-3.03 (m, $2 \mathrm{H}), 2.98-2.95(\mathrm{~m}, 1 \mathrm{H}), 2.88-2.84(\mathrm{~m}, 1 \mathrm{H}), 2.67-2.64(\mathrm{~m}, 1 \mathrm{H}), 2.38-2.28(\mathrm{~m}, 1 \mathrm{H}), 2.13-2.04(\mathrm{~m}, 1 \mathrm{H}), 1.32(\mathrm{~s}$, $18 \mathrm{H}), 1.11(\mathrm{~s}, 18 \mathrm{H}) \mathrm{ppm}$.

${ }^{13} \mathrm{C}$ NMR $\left(125 \mathrm{MHz}, \mathrm{CDCl}_{3}\right) \delta 167.27,167.19,154.56,154.45,150.65,150.05,148.31,148.16,136.33,133.76$, $133.60,130.43,129.41,129.16,129.14,128.20,127.63,126.52,125.89,125.62,125.34,124.98,123.63,123.46$, $123.63,123.46,123.39,121.13,121.00,120.85,120.04,119.82,119.30,117.58,115.29,112.89,112.40,71.44$, $71.15,70.91,70.58,70.47,70.18,69.89,68.79,68.72,68.64,63.44,47.99,43.88,42.35,34.97,34.63,31.48$, $27.00 \mathrm{ppm}$.

IR (KBr): $2962\left(v_{\mathrm{C}-\mathrm{H}}\right), 1710\left(v_{\mathrm{C}=\mathrm{O}, \text { ester }}\right), 1656\left(v_{\mathrm{C}=\mathrm{O} \text {, amide }}\right), 1248\left(v_{\mathrm{C}-\mathrm{O}-\mathrm{CO}, \mathrm{as}}\right), 1118\left(v_{\mathrm{C}-\mathrm{O}-\mathrm{CO}, \mathrm{s}}\right) \mathrm{cm}^{-1}$.

FAB-MS ( $m$-NBA): $m / z$ 1193.7, calcd for $[\mathrm{M}]^{+}: 1193.6$.

$[\alpha]_{\mathrm{D}}^{27} 192.2^{\circ}\left(\mathrm{c}=0.100, \mathrm{CH}_{2} \mathrm{Cl}_{2}\right)$.

Anal, calcd for $\mathrm{C}_{73} \mathrm{H}_{92} \mathrm{ClNO}_{11} \cdot \mathrm{H}_{2} \mathrm{O}: \mathrm{C}, 72.28 ; \mathrm{H}, 7.81 ; \mathrm{N}, 1.15$. found: $\mathrm{C}, 72.26 ; \mathrm{H}, 7.52 ; \mathrm{N}, 1.24$.

10b: amorphous solid. yield $92 \%$. m.p. $83-84^{\circ} \mathrm{C}$.

${ }^{1} \mathrm{H}$ NMR $\left(500 \mathrm{MHz}, \mathrm{CDCl}_{3}\right) \delta 7.94(\mathrm{~d}, J=9.0 \mathrm{~Hz}, 1 \mathrm{H}), 7.90(\mathrm{~d}, J=2.0 \mathrm{~Hz}, 2 \mathrm{H}), 7.86-7.82(\mathrm{~m}, 2 \mathrm{H}), 7.72(\mathrm{~d}, J=$ 8.0 Hz, 1H), 7.65-7.64 (m, 1H), 7.47-7.42 (m, 2H), 7.33-7.30 (m, 1H), 7.26-7.16 (m, 6H), 7.13 (s, 1H), 6.83-6.67 $(\mathrm{m}, 5 \mathrm{H}), 6.50(\mathrm{~s}, 2 \mathrm{H}), 4.59(\mathrm{~d}, J=16.0 \mathrm{~Hz}, 1 \mathrm{H}), 4.34-4.30(\mathrm{~m}, 1 \mathrm{H}), 4.20-4.03(\mathrm{~m}, 8 \mathrm{H}), 3.98(\mathrm{~d}, J=13.0 \mathrm{~Hz}, 1 \mathrm{H})$, 
3.93-3.89 (m, 1H), 3.83-3.69 (m, 4H), $3.61(\mathrm{~d}, J=16.0 \mathrm{~Hz}, 1 \mathrm{H}), 3.59-3.55(\mathrm{~m}, 2 \mathrm{H}), 3.49-3.34(\mathrm{~m}, 5 \mathrm{H}), 3.26-3.19$ $(\mathrm{m}, 4 \mathrm{H}), 3.12-3.09(\mathrm{~m}, 1 \mathrm{H}), 3.00-2.92(\mathrm{~m}, 2 \mathrm{H}), 2.84-2.81(\mathrm{~m}, 1 \mathrm{H}), 1.58-1.50(\mathrm{~m}, 2 \mathrm{H}), 1.37(\mathrm{~s}, 18 \mathrm{H}), 1.17(\mathrm{~s}, 18 \mathrm{H})$, 1.13-0.89 (m, 4H) ppm.

${ }^{13} \mathrm{C}$ NMR $\left(125 \mathrm{MHz}, \mathrm{CDCl}_{3}\right.$ ) $\delta 167.21,166.86,154.58,154.43,150.77,150.15,148.31,148.23,136.7,133.64$, $133.61,130.19,129.56,129.43,129.28,129.24,128.16,127.72,126.69,125.92,125.79,125.31,125.08,123.59$, $123.52,120.89,120.84,120.43,120.39,120.16,119.63,116.50,115.58,112.87,112.63,71.07,70.79,70.75$, $70.62,70.52,70.42,70.31,70.18,70.04,69.24,68.66,68.43,65.70,48.05,48.00,42.18,35.00,34.70,31.53$, $31.48,29.09,28.34,25.79,25.55 \mathrm{ppm}$.

IR (KBr): $2962\left(v_{\mathrm{C}-\mathrm{H}}\right), 1713\left(v_{\mathrm{C}=\mathrm{O}, \text { ester }}\right), 1654\left(v_{\mathrm{C}=\mathrm{O}, \text { amide }}\right), 1245\left(v_{\mathrm{C}-\mathrm{O}-\mathrm{CO}, \text { as }}\right), 1123\left(v_{\mathrm{C}-\mathrm{O}-\mathrm{CO}, \mathrm{s}}\right) \mathrm{cm}^{-1}$.

FAB-MS ( $m$-NBA): $m / z$ 1235.6, calcd for $[\mathrm{M}]^{+}: 1235.7$.

$[\alpha]_{\mathrm{D}}^{27} 191.8^{\circ}\left(\mathrm{c}=0.100, \mathrm{CH}_{2} \mathrm{Cl}_{2}\right)$.

Anal, calcd for $\mathrm{C}_{76} \mathrm{H}_{98} \mathrm{ClNO}_{11} \cdot \mathrm{H}_{2} \mathrm{O}: \mathrm{C}, 72.73 ; \mathrm{H}, 8.03 ; \mathrm{N}, 1.12$. found: $\mathrm{C}, 72.71 ; \mathrm{H}, 7.72 ; \mathrm{N}, 1.20$.

\section{(R)-Chiral thiazolium rotaxane (2)}

A mixture of 10a $(140 \mathrm{mg}, 0.117 \mathrm{mmol})$, sodium iodide $(175 \mathrm{mg}, 1.17 \mathrm{mmol})$, and thiazole $(0.70 \mathrm{~mL}, 9.8 \mathrm{mmol})$ was allowed to stand at $80{ }^{\circ} \mathrm{C}$ for $24 \mathrm{~h}$. The resulting mixture was poured into $1 \mathrm{M}$ hydrochloric acid $(100 \mathrm{~mL})$. The precipitate was collected by filtration. The crude product was dissolved in methanol and passed through anion exchange column (Amberlite ${ }^{\circledR}$ IRA-400 treated by hydrochloric acid followed by water, eluent: methanol). The crude product was further purified by preparative HPLC (eluent: chloroform).

2a: white powder. yield $52 \%$. m.p. $176-177^{\circ} \mathrm{C}$.

${ }^{1} \mathrm{H}$ NMR $\left(500 \mathrm{MHz}, \mathrm{CDCl}_{3}\right) \delta 9.95(\mathrm{br}, 1 \mathrm{H}), 8.30(\mathrm{~s}, 1 \mathrm{H}), 7.95(\mathrm{~d}, J=9.5 \mathrm{~Hz}, 1 \mathrm{H}), 7.89-7.85(\mathrm{~m}, 3 \mathrm{H}), 7.72(\mathrm{~s}$, $1 \mathrm{H}), 7.51(\mathrm{~d}, J=8.0 \mathrm{~Hz}, 1 \mathrm{H}), 7.38-7.13(\mathrm{~m}, 10 \mathrm{H}), 6.91-6.83(\mathrm{~m}, 3 \mathrm{H}), 6.66(\mathrm{~d}, J=8.0 \mathrm{~Hz}, 1 \mathrm{H}), 6.22(\mathrm{~s}, 2 \mathrm{H}), 5.96-$ $5.93(\mathrm{~m}, 1 \mathrm{H}), 4.75-4.61(\mathrm{~m}, 3 \mathrm{H}), 4.47-4.43(\mathrm{~m}, 1 \mathrm{H}), 4.25-4.20(\mathrm{~m}, 2 \mathrm{H}), 4.08-4.02(\mathrm{~m}, 4 \mathrm{H}), 3.92-3.6(\mathrm{~m}, 8 \mathrm{H})$, 3.57-3.02 (m, 10H), 2.30-2.84 (m, 1H), 2.64-2.60 (m, 1H), 2.33-2.18 (m, 4H), $1.38(\mathrm{~s}, 18 \mathrm{H}), 1.15(\mathrm{~s}, 18 \mathrm{H}) \mathrm{ppm}$.

${ }^{13} \mathrm{C}$ NMR $\left(125 \mathrm{MHz}, \mathrm{CDCl}_{3}\right) \delta 167.31,163.44,161.71,154.50,154.24,150.89,150.30,148.34,147.73,136.31$, $135.45,133.65,133.50,129.97,129.47,129.36,129.32,128.96,128.16,127.67,127.17,126.95,126.17,125.89$, $125.22,124.93,123.75,123.63,123.50,122.01,121.75,121.05,120.31,119.84,119.06,117.27,114.72,112.80$, $71.38,70.65,70.62,70.47,70.39,70.18,70.07,69.89,68.60,68.22,63.00,54.88,49.02,43.62,34.97,34.60$, $31.44,31.42,26.52 \mathrm{ppm}$.

IR (KBr): $2961\left(v_{\mathrm{C}-\mathrm{H}}\right), 1710\left(v_{\mathrm{C}=\mathrm{O}, \text { ester }}\right), 1662\left(v_{\mathrm{C}=\mathrm{O} \text {, amide }}\right), 1247\left(v_{\mathrm{C}-\mathrm{O}-\mathrm{CO}, \text { as }}\right), 1119\left(v_{\mathrm{C}-\mathrm{O}-\mathrm{CO}, \mathrm{s}}\right) \mathrm{cm}^{-1}$.

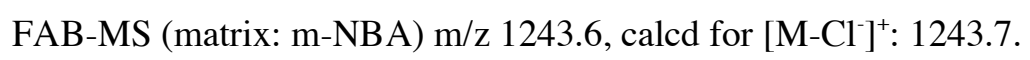

$[\alpha]_{\mathrm{D}}^{20} 245.6^{\circ}\left(\mathrm{c} 0.100, \mathrm{CH}_{2} \mathrm{Cl}_{2}\right)$.

Anal, calcd for $\mathrm{C}_{76} \mathrm{H}_{95} \mathrm{ClN}_{2} \mathrm{O}_{11} \mathrm{~S} \cdot 5 \mathrm{H}_{2} \mathrm{O}: \mathrm{C}, 66.62 ; \mathrm{H}, 7.72 ; \mathrm{N}, 2.04 ; \mathrm{S}, 2.34$. found: $\mathrm{C}, 66.88 ; \mathrm{H}, 7.36 ; \mathrm{N}, 2.18 ; \mathrm{S}$, 2.26 .

2b was similarly prepared using $\mathbf{1 0 b}(600 \mathrm{mg}, 0.485 \mathrm{mmol})$. The reaction was carried out at room temperature for $24 \mathrm{~h}$.

2b: white powder. yield $64 \%$. m.p. $142-143{ }^{\circ} \mathrm{C}$.

${ }^{1} \mathrm{H}$ NMR $\left(500 \mathrm{MHz}, \mathrm{CDCl}_{3}\right) \delta 9.88(\mathrm{br}, 1 \mathrm{H}), 8.19(\mathrm{~s}, 1 \mathrm{H}), 7.91-7.90(\mathrm{~m}, 4 \mathrm{H}), 7.86(\mathrm{~d}, J=8.0 \mathrm{~Hz}, 1 \mathrm{H}), 7.78(\mathrm{~d}, J=$ $8.5 \mathrm{~Hz}, 1 \mathrm{H}), 7.66(\mathrm{~s}, 1 \mathrm{H}), 7.50(\mathrm{~d}, J=9.0 \mathrm{~Hz}, 1 \mathrm{H}), 7.36-7.15(\mathrm{~m}, 9 \mathrm{H}), 6.87-6.85(\mathrm{~m}, 3 \mathrm{H}), 6.69(\mathrm{~d}, J=7.5 \mathrm{~Hz}, 1 \mathrm{H})$, $6.37(\mathrm{~s}, 2 \mathrm{H}), 5.77(\mathrm{~d}, J=17.5 \mathrm{~Hz}, 1 \mathrm{H}), 4.83(\mathrm{~d}, J=16.5 \mathrm{~Hz}, 1 \mathrm{H}), 4.50-4.21(\mathrm{~m}, 2 \mathrm{H}), 4.27-4.07(\mathrm{~m}, 5 \mathrm{H}), 4.01-3.92$ $(\mathrm{m}, 3 \mathrm{H}), 3.84-3.69(\mathrm{~m}, 4 \mathrm{H}), 3.62-3.46(\mathrm{~m}, 7 \mathrm{H}), 3.33-3.20(\mathrm{~m}, 4 \mathrm{H}), 3.13-3.12(\mathrm{~m}, 2 \mathrm{H}), 2.97-2.93 .(\mathrm{m}, 1 \mathrm{H}), 2.69-$ $2.65(\mathrm{~m}, 1 \mathrm{H}), 2.39$ (br, 3H), 1.66-1.60 (m, 4H), $1.36(\mathrm{~s}, 18 \mathrm{H}), 1.26-1.23(\mathrm{~m}, 2 \mathrm{H}), 1.18(\mathrm{~s}, 18 \mathrm{H}) \mathrm{ppm}$.

${ }^{13} \mathrm{C} \mathrm{NMR}\left(125 \mathrm{MHz}, \mathrm{CDCl}_{3}\right) \delta 167.20,163.27,161.24,154.48,154.26,150.88,150.21,148.23,147.96,136.38$, 
$135.85,133.46,133.39,129.81,129.51,129.49,129.43,129.03,128.05,127.68,126.91,126.53,126.24,126.03$, $125.18,124.96,123.81,123.78,123.46,121.78,121.43,120.43,120.29,120.20,119.25,116.04,115.22,113.95$, $112.78,70.99,70.82,70.52,70.51,70.47,70.38,70.31,70.13,69.45,68.79,68.62,65.07,55.16,49.05,47.53$, $34.93,34.60,31.43,31.40,29.41,27.69,25.95,25.89 \mathrm{ppm}$.

IR (KBr): $2961\left(v_{\mathrm{C}-\mathrm{H}}\right), 1714\left(v_{\mathrm{C}=\mathrm{O} \text {, ester }}\right), 1658\left(v_{\mathrm{C}=\mathrm{O} \text {, amide }}\right), 1245\left(v_{\mathrm{C}-\mathrm{O}-\mathrm{CO}, \text { as }}\right), 1124\left(v_{\mathrm{C}-\mathrm{O}-\mathrm{CO}, \mathrm{s}}\right) \mathrm{cm}^{-1}$.

FAB-MS ( $m$-NBA): $m / z$ 1285.7, calcd for $\left[\mathrm{M}-\mathrm{Cl}^{-}\right]^{+}: 1285.7$.

$[\alpha]_{\mathrm{D}}^{20} 230.0^{\circ}\left(\mathrm{c} 0.100, \mathrm{CH}_{2} \mathrm{Cl}_{2}\right)$.

Anal, calcd for $\mathrm{C}_{79} \mathrm{H}_{101} \mathrm{ClN}_{2} \mathrm{O}_{11} \mathrm{~S} \cdot 4 \mathrm{H}_{2} \mathrm{O}: \mathrm{C}, 68.05 ; \mathrm{H}, 7.88 ; \mathrm{N}, 2.01 ; \mathrm{S}, 2.30$. found: $\mathrm{C}, 68.17 ; \mathrm{H}, 7.56 ; \mathrm{N}, 2.14 ; \mathrm{S}$, 2.20 .

\section{Scheme $\mathbf{S 2}$}

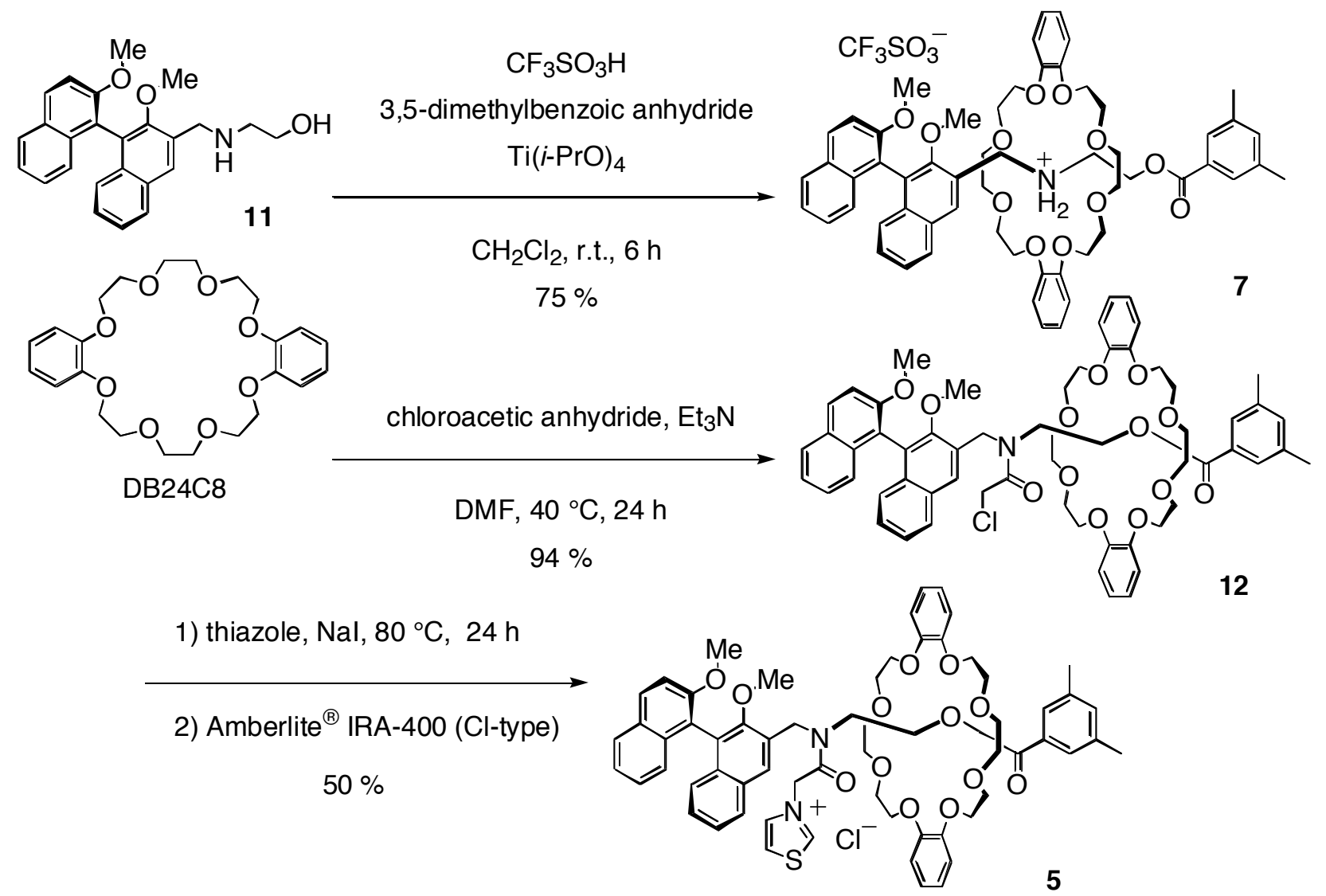

\section{(R)-Chiral rotaxane (7)}

To a mixture of $(R)$-aminoalcohol $11(327 \mathrm{mg}, 0.84 \mathrm{mmol})$, DB24C8 $(397 \mathrm{mg}, 0.89 \mathrm{mmol})$, and titanium isopropoxide $(2.5 \mu \mathrm{L}, 8.4 \mathrm{mmol})$ were added dichloromethane $(1.2 \mathrm{~mL})$ and triflic acid $(112 \mu \mathrm{L}, 1.27 \mathrm{mmol})$ at $0{ }^{\circ} \mathrm{C}$ under an argon atmosphere. The mixture was stirred at $0{ }^{\circ} \mathrm{C}$ for $30 \mathrm{~min}$. 3,5-Dimethylbenzoic anhydride (298 $\mathrm{mg}, 1.06 \mathrm{mmol}$ ) was added to the solution. After the reaction mixture was carried out at room temperature for $6 \mathrm{~h}$, the reaction mixture was washed with $5 \%$ sodium carbonate solution, $1 \mathrm{M}$ hydrochloric acid, then brine, dried over anhydrous magnesium sulfate, and evaporated in vacuo to give a crude product, which was purified by silica gel column chromatography (eluent: chloroform/methanol $=100 / 1)$ to give $702 \mathrm{mg}(75 \%)$ of 7 as a white amorphous solid.

m.p. $96-98^{\circ} \mathrm{C}$.

${ }^{1} \mathrm{H}$ NMR (500 MHz, $\left.\mathrm{CDCl}_{3}\right) \delta 8.03(\mathrm{~d}, J=9.5 \mathrm{~Hz}, 1 \mathrm{H}), 7.88(\mathrm{~s}, 1 \mathrm{H}), 7.85(\mathrm{~d}, J=8.0 \mathrm{~Hz}, 1 \mathrm{H}), 7.73(\mathrm{br}, 2 \mathrm{H}), 7.64$ (s, 2H), 7.53 (d, $J=8.5 \mathrm{~Hz}, 1 \mathrm{H}), 7.47(\mathrm{dd}, J=1.8,9.3 \mathrm{~Hz}, 1 \mathrm{H}), 7.33-7.27(\mathrm{~m}, 2 \mathrm{H}), 7.19-7.02(\mathrm{~m}, 5 \mathrm{H}), 6.85$ (br, $8 \mathrm{H}), 4.74$ (br, 2H), 4.65-4.59 (m, 2H), 4.20-4.05 (m, 10H), 3.82 (br, 8H), 3.75 (s, 3H), 3.70-3.64 (m, 8H), 3.21 (s, 
$3 \mathrm{H}), 2.17$ (s, 6H) ppm.

${ }^{13} \mathrm{C}$ NMR $\left(125 \mathrm{MHz}, \mathrm{CDCl}_{3}\right) \delta 165.88,154.43,153.46,147.01,137.55,134.67,134.19,133.20,130.35,130.01$, $129.25,128.51,127.69,127.66,126.96,126.81,126.06,124.89,124.66,124.32,123.78,123.62,123.25,121.32$, $121.29,120.61\left(\mathrm{q}, J_{\mathrm{C}-\mathrm{F}}=320 \mathrm{~Hz}\right), 117.51,112.95,112.32,112.30,77.20,70.53,70.48,69.93,69.88,67.89,67.82$, 60.46, 60.07, 55.96, 48.87, 47.92, $20.64 \mathrm{ppm}$.

IR (KBr): $2930\left(v_{\mathrm{C}-\mathrm{H}}\right), 1720\left(v_{\mathrm{C}=\mathrm{O}}\right.$ ester $), 1266\left(v_{\mathrm{S}=\mathrm{O}, \text { as }}\right), 1031\left(v_{\mathrm{S}=\mathrm{O}, \mathrm{s}}\right), 637\left(v_{\mathrm{S}-\mathrm{O}}\right) \mathrm{cm}^{-1}$.

FAB-MS ( $m$-NBA): $m / z, 968.5$, calcd for $\left[\mathrm{M}-\mathrm{CF}_{3} \mathrm{SO}_{3}{ }^{-}\right]^{+}: 968.5$.

$[\alpha]_{\mathrm{D}}^{26} 38.0^{\circ}\left(\mathrm{c}=0.100, \mathrm{CH}_{2} \mathrm{Cl}_{2}\right)$.

Anal, calcd for $\mathrm{C}_{59} \mathrm{H}_{66} \mathrm{~F}_{3} \mathrm{NO}_{15} \mathrm{~S} \cdot \mathrm{H}_{2} \mathrm{O}: \mathrm{C}, 62.37 ; \mathrm{H}, 6.03 ; \mathrm{N}, 1.23$. found: $\mathrm{C}, 62.27 ; \mathrm{H}, 6.09 ; \mathrm{N}, 1.36$.

\section{(R)-N-chloroacetyl rotaxane (12)}

A solution of $7(1.16 \mathrm{~g}, 1.04 \mathrm{mmol})$, chloroacetic anhydride $(1.77 \mathrm{~g}, 10.4 \mathrm{mmol})$, and triethylamine $(724 \mu \mathrm{L}, 5.19$ mmol) in $N, N$-dimethylformamide $(15 \mathrm{~mL})$ was stirred at $40{ }^{\circ} \mathrm{C}$ for $24 \mathrm{~h}$. After addition of $5 \%$ aqueous sodium carbonate solution, the reaction mixture was extracted with chloroform. The organic layer was washed with a mixture of $5 \%$ aqueous sodium carbonate solution and methanol ( $/ \mathrm{v}=1 / 1), 2 \mathrm{M}$ hydrochloric acid, then brine, dried over anhydrous magnesium sulfate, and evaporated in vacuo to give a crude product, which was purified by silica gel column chromatography (eluent: chloroform) to give $1.02 \mathrm{~g}(94 \%)$ of $\mathbf{1 2}$ as a white solid.

m.p. $214-215^{\circ} \mathrm{C}$.

${ }^{1} \mathrm{H}$ NMR $\left(500 \mathrm{MHz}, \mathrm{CDCl}_{3}\right) \delta 8.06(\mathrm{~s}, 2 \mathrm{H}), 7.97(\mathrm{~d}, J=9.5 \mathrm{~Hz}, 1 \mathrm{H}), 7.84(\mathrm{~d}, J=8.0 \mathrm{~Hz}, 1 \mathrm{H}), 7.43-7.40(\mathrm{~m}, 2 \mathrm{H})$, 7.29-7.25 (m, 2H), 7.13-7.07 (m, 5H), $6.97(\mathrm{~d}, J=8.0 \mathrm{~Hz}, 1 \mathrm{H}), 6.85-6.69(\mathrm{~m}, 8 \mathrm{H}), 5.25-5.20(\mathrm{~m}, 1 \mathrm{H}), 5.13-5.07$ $(\mathrm{m}, 1 \mathrm{H}), 4.66-4.52(\mathrm{~m}, 2 \mathrm{H}), 4.47-4.34(\mathrm{~m}, 2 \mathrm{H}), 4.26-4.22(\mathrm{~m}, 3 \mathrm{H}), 4.11-4.08(\mathrm{~m}, 3 \mathrm{H}), 3.96-3.78(\mathrm{~m}, 8 \mathrm{H}), 3.71(\mathrm{~s}$, $3 \mathrm{H}), 3.68-3.64(\mathrm{~m}, 2 \mathrm{H}), 3.55-3.39(\mathrm{~m}, 10 \mathrm{H}), 3.16(\mathrm{~s}, 3 \mathrm{H}), 2.27$ (s, 6H) ppm.

${ }^{13} \mathrm{C}$ NMR $\left(125 \mathrm{MHz}, \mathrm{CDCl}_{3}\right) \delta 167.54,166.48,154.70,154.43,148.23,148.11,137.35,133.99,133.56,132.77$, $131.10,130.67,130.40,129.37,128.93,128.78,128.04,127.68,125.85,125.37,124.99,124.54,124.50,124.02$, $123.61,123.28,120.57,120.50,119.65,113.40,112.56,111.86,71.26,71.19,70.30,70.26,68.06,68.05,62.30$, $60.05,56.22,45.70,44.35,42.66,21.14 \mathrm{ppm}$.

IR (KBr): $2918\left(v_{\mathrm{C}-\mathrm{H}}\right), 1709\left(v_{\mathrm{C}=\mathrm{O} \text {, ester }}\right), 1658\left(v_{\mathrm{C}=\mathrm{O} \text {, amide }}\right), 1248\left(v_{\mathrm{C}-\mathrm{O}-\mathrm{CO}, \text { as }}\right), 1126\left(v_{\mathrm{C}-\mathrm{O}-\mathrm{CO}, \mathrm{s}}\right) \mathrm{cm}^{-1}$.

FAB-MS ( $m$-NBA): $m / z \quad 1043.4$, calcd for $[\mathrm{M}]^{+}: 1043.4$.

$[\alpha]_{\mathrm{D}}^{26} 42.4^{\circ}\left(\mathrm{c}=0.100, \mathrm{CH}_{2} \mathrm{Cl}_{2}\right)$.

Anal, calcd for $\mathrm{C}_{60} \mathrm{H}_{66} \mathrm{ClNO}_{13} \cdot \mathrm{H}_{2} \mathrm{O}: \mathrm{C}, 67.82 ; \mathrm{H}, 6.45 ; \mathrm{N}, 1.32$. found: $\mathrm{C}, 68.06 ; \mathrm{H}, 6.63 ; \mathrm{N}, 1.47$.

\section{$(R)$-chiral thiazolium rotaxane (5)}

A mixture of 12 (486 mg, $0.48 \mathrm{mmol})$, sodium iodide $(672 \mathrm{mg}, 4.48 \mathrm{mmol})$, and thiazole (1.0 $\mathrm{mL}, 14 \mathrm{mmol})$ was allowed to stand at $80{ }^{\circ} \mathrm{C}$ for $48 \mathrm{~h}$. The resulting mixture was poured into $1 \mathrm{M}$ hydrochloric acid $(100 \mathrm{~mL})$. The precipitate was collected by filtration. The crude products were dissolved in methanol, and the solution was passed through anion exchange column (Amberlite ${ }^{\circledR}$ IRA-400 treated by hydrochloric acid followed by methanol, eluent: methanol). The crude product was further purified by silica gel column chromatography (eluent: chloroform/methanol $=10 / 1)$ to give $281 \mathrm{mg}(56 \%)$ of 5 as white powder.

m.p. $172{ }^{\circ} \mathrm{C} .(\mathrm{dec}$.

${ }^{1} \mathrm{H}$ NMR (500 MHz, $\mathrm{CDCl}_{3}$ ) $\delta 10.75(\mathrm{br}, 1 \mathrm{H}), 8.17$ (br, 1H), 7.98-7.96 (m, 2H), $7.92(\mathrm{~s}, 2 \mathrm{H}), 7.84(\mathrm{~d}, J=8.5 \mathrm{~Hz}$, $1 \mathrm{H}), 7.61(\mathrm{~d}, J=8.0 \mathrm{~Hz}, 1 \mathrm{H}), 7.41(\mathrm{~d}, J=9.0 \mathrm{~Hz}, 1 \mathrm{H}), 7.30-7.26(\mathrm{~m}, 2 \mathrm{H}), 7.22(\mathrm{~s}, 1 \mathrm{H}), 7.13-6.98(\mathrm{~m}, 5 \mathrm{H}), 6.82$ (br, 6H), $6.76(\mathrm{br}, 2 \mathrm{H}), 6.16(\mathrm{~d}, J=16.0 \mathrm{~Hz}, 1 \mathrm{H}), 5.92(\mathrm{~d}, J=16.0 \mathrm{~Hz}, 1 \mathrm{H}), 5.32-5.26(\mathrm{~m}, 1 \mathrm{H}), 5.17-5.11(\mathrm{~m}, 1 \mathrm{H})$, $4.68(\mathrm{~s}, 2 \mathrm{H}), 4.47-4.35(\mathrm{~m}, 2 \mathrm{H}), 4.26-4.23(\mathrm{~m}, 2 \mathrm{H}), 4.13-4.03(\mathrm{~m}, 6 \mathrm{H}), 3.95-3.89(\mathrm{~m}, 4 \mathrm{H}), 3.68(\mathrm{~s}, 3 \mathrm{H}), 3.65-3.50$ 
(m, 12H), $3.12(\mathrm{~s}, 3 \mathrm{H}), 2.27$ (s, 6H) ppm.

${ }^{13} \mathrm{C} \mathrm{NMR}\left(125 \mathrm{MHz}, \mathrm{CDCl}_{3}\right) \delta 166.60,163.98,162.03,154.64,154.21,148.21,137.50,133.88,133.83,132.89$, $130.56,130.32$, 129.85, 129.55, 128.90, 128.57, 127.74, 125.99, 125.40, 125.30, 125.17, 124.66, 124.39, 124.26, $124.11,123.34,121.26,121.14,119.20,113.42,113.39,112.92,71.32,71.24,70.41,68.86,68.73,61.86,60.00$, $56.25,55.64,45.80,45.10,21.13 \mathrm{ppm}$.

IR (KBr): $2919\left(v_{\mathrm{C}-\mathrm{H}}\right), 1709\left(v_{\mathrm{C}=\mathrm{O}, \text { ester }}\right), 1664\left(v_{\mathrm{C}=\mathrm{O}, \text { amide }}\right), 1248\left(v_{\mathrm{C}-\mathrm{O}-\mathrm{CO}, \text { as }}\right), 1125\left(v_{\mathrm{C}-\mathrm{O}-\mathrm{CO}, \mathrm{s}}\right) \mathrm{cm}^{-1}$.

FAB-MS (matrix: m-NBA) m/z 1093.1, calcd for [M-Cl- $]^{+}:$1093.5.

$[\alpha]_{\mathrm{D}}^{29} 25.4^{\circ}\left(\mathrm{c}=0.100, \mathrm{CH}_{2} \mathrm{Cl}_{2}\right)$.

Anal, calcd for $\mathrm{C}_{63} \mathrm{H}_{69} \mathrm{ClN}_{2} \mathrm{O}_{13} \mathrm{~S} \cdot 4 \mathrm{H}_{2} \mathrm{O}: \mathrm{C}, 62.96 ; \mathrm{H}, 6.46 ; \mathrm{N}, 2.33 ; \mathrm{S}, 2.67$. found: $\mathrm{C}, 62.61 ; \mathrm{H}, 6.24 ; \mathrm{N}, 2.48 ; \mathrm{S}$, 2.65 .

\section{references}

1) Demir, A. S.; Sesenoglu, Ö.; Eren, E; Hosrik, B.; Pohl, M.; Janzen, E.; Kolter, D.; Feldmann, R.; Dünkelmann, P.; Müller, M. Adv. Synth. Catal. 2002, 344, 96. 